\title{
TABLE OF PRIMITIVE BINARY POLYNOMIALS. II
}

\author{
MIODRAG ŽIVKOVIĆ
}

\begin{abstract}
For those $n<5000$, for which the factorization of $2^{n}-1$ is known, the first primitive trinomial (if such exists) and a randomly generated primitive 5- and 7-nomial of degree $n$ in $\mathrm{GF}(2)$ are given, if the respective entry is absent from the previously published table.
\end{abstract}

After our paper with a list of primitive binary polynomials [3] had been accepted for publication, S. S. Wagstaff, Jr. made available to us reference [2] with some new factorizations of $2^{n}-1$ (updates to [1] obtained up to April $5,1993)$. This enabled us to make an update to our list, in which the smallest degree of missing primitive binary polynomial is now 511 .

Here, like in our previous table, the degrees of nonzero coefficients (except 0 and $n$ ) of primitive $k$-nomials (if there are any) of degree $n$ are given for some missing $2 \leq n \leq 5000$.

TABLE 1. Primitive binary polynomials

\begin{tabular}{|c|c|c|c|c|c|c|c|c|c|}
\hline$n \backslash k$ & 3 & $\longleftarrow$ & 5 & $\longrightarrow$ & $\longleftarrow$ & - & 7 & - & $\longrightarrow$ \\
\hline 311 & & 26 & 77 & 206 & 117 & 127 & 233 & 245 & \\
\hline 349 & & 122 & 289 & 299 & 52 & 89 & 179 & 212 & 240 \\
\hline 353 & 69 & 172 & 234 & 329 & 107 & 109 & 186 & 200 & 29 \\
\hline 361 & & 18 & 40 & 227 & 7 & 82 & 179 & 217 & 24 \\
\hline 367 & 21 & 136 & 332 & 335 & 59 & 174 & 261 & 270 & 286 \\
\hline 383 & 90 & 270 & 292 & 380 & 104 & 132 & 218 & 225 & 287 \\
\hline 391 & 28 & 202 & 231 & 316 & 5 & 35 & 45 & 213 & 378 \\
\hline 397 & & 41 & 184 & 302 & 21 & 171 & 182 & 254 & 73 \\
\hline 401 & 152 & 163 & 224 & 345 & 136 & 142 & 186 & 277 & 20 \\
\hline 407 & 71 & 106 & 236 & 402 & 88 & 218 & 275 & 358 & 59 \\
\hline 413 & & 151 & 152 & 232 & 135 & 165 & 227 & 244 & 440 \\
\hline 419 & & 38 & 120 & 382 & 10 & 178 & 219 & 248 & 66 \\
\hline 423 & 25 & 61 & 181 & 3 & 45 & 58 & 145 & 310 & 85 \\
\hline 445 & & 129 & 255 & 420 & 39 & 198 & 208 & 363 & 433 \\
\hline 449 & 134 & 263 & 285 & J & 9 & 104 & 222 & 243 & 429 \\
\hline 463 & 93 & 151 & 15 & 25 & 100 & 191 & 226 & 293 & 08 \\
\hline 467 & & 72 & 10 & 3 & 60 & 154 & 326 & 336 & 45 \\
\hline 473 & & 73 & 155 & 304 & 157 & 197 & 239 & 275 & 70 \\
\hline
\end{tabular}

Received by the editor June 11, 1993.

1991 Mathematics Subject Classification. Primary 11T06, 11 T71.

Key words and phrases. Primitive polynomials, finite field.

This research was supported by Science Fund of Serbia, grant number 0401A, through Matematički institut. 
TABLE 1 (continued)

\begin{tabular}{|c|c|c|c|c|c|c|c|c|c|}
\hline$n \backslash k$ & 3 & $\longleftarrow$ & 5 & $\longrightarrow$ & $\longleftarrow$ & - & 7 & - & $\longrightarrow$ \\
\hline 475 & & & 104 & 178 & 46 & & 363 & & \\
\hline 479 & 104 & 103 & 245 & 270 & 54 & 127 & 164 & 359 & \\
\hline 481 & 138 & 342 & 442 & 446 & 94 & 206 & 268 & 318 & \\
\hline 485 & & 28 & 129 & 463 & 195 & 197 & 238 & 367 & 99 \\
\hline 491 & & 137 & 150 & 248 & 15 & 58 & 50 & 216 & \\
\hline 499 & & 115 & 154 & 403 & 56 & 110 & 51 & 322 & \\
\hline 501 & & 49 & 437 & 499 & 24 & 142 & 48 & 81 & \\
\hline 515 & & 123 & 328 & 418 & 96 & 171 & 91 & 10 & 78 \\
\hline 517 & & 105 & 290 & 391 & 23 & 157 & 06 & 259 & 45 \\
\hline 523 & & 156 & 245 & 460 & 100 & 243 & 290 & 293 & 52 \\
\hline 535 & & 70 & 95 & 282 & & 114 & 267 & 74 & 6 \\
\hline 541 & & 185 & 407 & 426 & 148 & 176 & 234 & 325 & 07 \\
\hline 545 & 122 & 17 & 437 & 504 & 71 & 258 & 353 & 360 & 05 \\
\hline 587 & & 30 & 52 & 24 & 92 & 125 & 19 & 33 & 91 \\
\hline 622 & 297 & 72 & 488 & 58 & 29 & 62 & 07 & 6/ & 04 \\
\hline 627 & & 200 & 225 & 51 & 496 & 539 & 557 & בנד & 63 \\
\hline 633 & 101 & 100 & 101 & 418 & 19 & 171 & 182 & 90 & 2 \\
\hline 637 & & 342 & 588 & 595 & 285 & 422 & 426 & 486 & \\
\hline 63 & 16 & 15 & 249 & 439 & 17 & 414 & 447 & 497 & 23 \\
\hline 6 & & 167 & 452 & 466 & 122 & 202 & 250 & 347 & 03 \\
\hline 649 & 37 & 253 & 48 & 61 & 83 & 05 & 0 & 31 & 88 \\
\hline 651 & & 58 & 21 & 64 & 143 & 160 & 50 & 35 & 72 \\
\hline 65. & & 178 & 3. & 37 & 64 & 294 & 1 & 3 & 80 \\
\hline 66 & & 219 & 249 & 649 & 259 & 14 & 2 & 12 & 1 \\
\hline & & 182 & $\sigma_{1}$ & 51 & 47 & 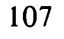 & 8 & 90 & 67 \\
\hline 67 & & 67 & 115 & 18 & 231 & 6 & 3 & 2 & 95 \\
\hline 6 & & 61 &  & 4. & 169 &  & 1 & 31 & 92 \\
\hline 6 & 13 & 41 & 12 & 5 & 70 & 140 & 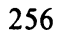 & 30 & 58 \\
\hline 6 & 215 & 9 & 416 & 53 & 45 & 199 & 368 & 04 & 50 \\
\hline 70 & 19 & 57 & 1 & 66 & 54 & 15 & $3 / 8$ & 424 & 6 \\
\hline 70 & & 130 & & 2 & 44 & 149 & 39 & 9 & 2 \\
\hline 72 & 231 & 95 & 1 & 2 & 109 & 4 & 460 & 511 & 68 \\
\hline 73 & & 79 &  & 6 & 11 & " & 156 & 7 & 52 \\
\hline 73 & & 289 & 6 & 68 & 35 & 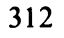 & 416 & 418 & 7 \\
\hline 74 & & 86 & 3 & 62 & 266 & 285 & $4 / 6$ & 01 & 2 \\
\hline 74 & 351 & 119 & & & & 208 & 2 & 73 & \\
\hline & & 70 & & 5 & & 03 & & & 1 \\
\hline 76 & & 6 & 1 & 47 & 140 & 197 & 5 & 462 & 8 \\
\hline 765 & & 398 & 41 & 67 & 2 & 299 & 2 & 609 & 72 \\
\hline 766 & & 154 & 269 & 48 & 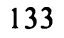 & 292 & 6 & 54 & 9 \\
\hline 765 & 120 & 31 & 2 & 4 & 128 & 28 & 19 & 1 & 50 \\
\hline 77 & 23 & 113 & & & 59 & & & 36 & 4 \\
\hline 78 & 329 & 75 & & & 275 & 0 & 3 & 619 & 9 \\
\hline 78 & & 3 & 2 & & 123 & & 3 & 598 & 1 \\
\hline 79 & 14 & 256 & 4 & 5 & 18 & 49 & 5 & 683 & 8 \\
\hline 80 & 217 & 269 & 409 & 54 & 289 & 500 & 575 & 590 & 3 \\
\hline 802 & & 362 & 535 & 78 & 11 & 159 & 190 & 226 & 4 \\
\hline 808 & & 209 & 258 & 32 & 192 & 497 & 500 & 581 & 80 \\
\hline 813 & & 9 & 542 & 773 & 75 & 181 & 225 & 578 & 0 \\
\hline 814 & 145 & 197 & 334 & 6 & 237 & 307 & 316 & 515 & 5 \\
\hline 818 & 119 & 547 & 640 & 80 & & 113 & 226 & 480 & 1 \\
\hline 819 & & 1 & 126 & 69 & 59 & 394 & 421 & 593 & 9 \\
\hline 82 & 38 & 443 & 458 & 57 & 523 & 545 & 680 & 682 & 71 \\
\hline 82 & 255 & 227 & 333 & 616 & 55 & 291 & 341 & 446 & 51 \\
\hline 838 & 61 & 446 & 527 & 715 & 51 & 280 & 435 & 515 & \\
\hline 846 & & 137 & 356 & 780 & 32 & 34 & 89 & 269 & \\
\hline
\end{tabular}


TABLE OF PRIMITIVE BINARY POLYNOMIALS. II

TABLE 1 (continued)

\begin{tabular}{|c|c|c|c|c|c|c|c|c|c|}
\hline$n \backslash k$ & 3 & $\longleftarrow$ & 5 & $\longrightarrow$ & $\longleftarrow$ & - & 7 & - & $\longrightarrow$ \\
\hline & & & & & & & & & \\
\hline 84 & 13 & & & 3 & $\begin{array}{r}21 \\
260\end{array}$ & $1 / 11$ & 3 & 2 & \\
\hline 851 & & $39 \hat{~}$ & 66 & 700 & 130 & 88 & 52 & 0 & \\
\hline 85 & & 346 & 429 & 793 & 135 & 429 & 178 & 640 & 53 \\
\hline 856 & & 249 & 451 & 780 & 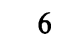 & 192 & 482 & 629 & 14 \\
\hline 869 & & 146 & 304 & 837 & 294 & 379 & 447 & 550 & \\
\hline 872 & & 257 & 480 & 507 & 197 & 382 & 489 & 615 & 57 \\
\hline 874 & & 39 & 545 & 820 & 37 & 117 & 494 & 596 & 27 \\
\hline 875 & & 445 & 469 & 576 & 84 & 220 & 423 & 658 & 49 \\
\hline 878 & & 180 & 488 & 725 & 200 & 261 & 493 & 693 & 62 \\
\hline 879 & 11 & 47 & 514 & 722 & 35 & 41 & 135 & 456 & 42 \\
\hline 880 & & 427 & 593 & 741 & 101 & 373 & 462 & 635 & 00 \\
\hline 885 & & 495 & 631 & 741 & & 37 & 37 & 367 & 754 \\
\hline 886 & & 29 & 304 & 4 & 95 & 227 & 376 & 708 & 795 \\
\hline 890 & & 31 & 479 & 525 & 194 & 377 & 449 & 626 & 97 \\
\hline 891 & & 131 & 182 & 523 & 1 & 553 & 638 & 811 & 56 \\
\hline 898 & 207 & 109 & 516 & 888 & 213 & 257 & 626 & 798 & 35 \\
\hline 901 & & 94 & 119 & 693 & 100 & 155 & 188 & 286 & 675 \\
\hline $90^{\circ}$ & & 94 & 558 & 583 & 133 & 13 & 228 & 552 & 758 \\
\hline 914 & & 293 & 534 & 860 & 14 & 104 & 207 & 64 & 15 \\
\hline 920 & & 65 & 264 & 475 & 126 & 379 & 499 & 624 & 851 \\
\hline 921 & 221 & 320 & 494 & 88 & 52 & 366 & 599 & 711 & 904 \\
\hline 922 & & 231 & 508 & 54 & 73 & 612 & 635 & 712 & 35 \\
\hline 926 & 365 & 126 & 657 & 914 & 1 & 203 & 273 & 302 & 65 \\
\hline 928 & & 157 & 394 & 54 & 162 & 25 & 483 & 732 & 00 \\
\hline 934 & & 510 & 565 & 7 & 89 & 228 & 374 & 480 & 50 \\
\hline 938 & 207 & 263 & 535 & 8 & 61 & 326 & 37 & 501 & 767 \\
\hline 944 & & 305 & 678 & 689 & 238 & 70 & 357 & 417 & 06 \\
\hline 946 & & 49 & 213 & 6 & 56 & 193 & 249 & 273 & 606 \\
\hline 950 & & 525 & 4 & & 62 & 77 & 56 & 60 & 42 \\
\hline 93 & & 44 & 490 & 6 & 208 & 52 & 431 & 566 & 11 \\
\hline 958 & & 8 & 540 & 5 & 144 & 363 & 779 & 873 & 90 \\
\hline 962 & & 129 & 302 & 4 & 5 & 203 & 334 & 359 & 936 \\
\hline 965 & & 12 & 06 & 437 & 1 & 175 & 374 & 07 & 25 \\
\hline 966 & & 81 & 6 & 850 & 112 & 219 & 9 & 37 & 893 \\
\hline 96 & & 51 & 0 & 90 & 3 & & 5 & 678 & 19 \\
\hline 970 & & 398 & 417 & 43 & 211 & 620 & 670 & 710 & 952 \\
\hline 974 & & 130 & 556 & 85 & 235 & 314 & 504 & 737 & 791 \\
\hline 975 & 19 & 246 & 925 & 949 & 295 & 335 & 394 & 418 & 36 \\
\hline 97 & & 13 & 195 & 4 & 12 & 273 & 372 & 23 & 4 \\
\hline 982 & 277 & 118 & 200 & 30 & 110 & 19 & 26 & 299 & 853 \\
\hline 986 & & 456 & 672 & 85 & 177 & 214 & 317 & 757 & 899 \\
\hline 987 & & 296 & 506 & 78 & 206 & 230 & 284 & 756 & 849 \\
\hline 992 & & 469 & 635 & 750 & 62 & 484 & 536 & 896 & 943 \\
\hline 99. & 223 & 459 & 529 & 978 & 182 & 278 & 336 & 919 & 938 \\
\hline 98 & 101 & 97 & 653 & 736 & 40 & 50 & 489 & 654 & 901 \\
\hline 10 & & 27 & 452 & 58 & 80 & 363 & 607 & 895 & 98 \\
\hline & & 343 & 737 & 90 & 93 & 12 & 139 & 858 & 95 \\
\hline & & 14 & 512 & 56 & 47 & 53 & 639 & 705 & 992 \\
\hline & & 558 & 751 & 10 & 127 & 27 & 368 & 524 & 38 \\
\hline & 93 & 93 & 117 & 100 & 54 & 109 & 149 & 390 & 816 \\
\hline 1( & 75 & 7 & 390 & 69 & 287 & 288 & 378 & 843 & 974 \\
\hline & & 95 & 138 & 903 & 310 & 611 & 647 & 681 & 76 \\
\hline
\end{tabular}


TABLE 1 (continued)

\begin{tabular}{|c|c|c|c|c|c|c|c|c|c|}
\hline$n \backslash k$ & 3 & 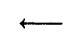 & 5 & $\longrightarrow$ & 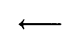 &  & & & \\
\hline & & & & & & & & & \\
\hline 1041 & 412 & 108 & 167 & 515 & 374 & 661 & 764 &  & \\
\hline 1046 & & 164 & 297 & 301 & 354 & 365 & 592 & 836 & \\
\hline 10 & 10 & 15 & 795 & 869 & 115 & 701 & 723 & 741 & \\
\hline 1053 & & 405 & 454 & 801 & 99 & 478 & 585 & 615 & \\
\hline 1057 & 198 & J & 122 & 1007 & 187 & 559 & 655 & 787 & \\
\hline 1062 & & 123 & 183 & 917 & 133 & 206 & 397 & 529 & \\
\hline 1074 & & 703 & 1048 & 1066 & 132 & 316 & 331 & 738 & 100 \\
\hline 1082 & 407 & 82 & 199 & 278 & 3 & 82 & 91 & 535 & \\
\hline 1090 & 79 & 167 & 578 & 808 & 656 & 766 & 84 & 941 & \\
\hline 109 & 261 & 312 & 645 & 1080 & 128 & 189 & & 553 & \\
\hline 110 & 65 & J & 178 & 925 & 661 & 771 & 800 & 862 & \\
\hline 1107 & & 380 & 577 & 823 & 19 & 129 & 184 & 257 & \\
\hline 1120 & & 294 & 718 & 865 & 165 & 285 & 456 & 568 & \\
\hline 1128 & & 113 & 154 & 577 & 121 & 148 & 163 & 255 & \\
\hline 1154 & 75 & 510 & 953 & 1105 & 83 & 729 & 766 & 771 & 102 \\
\hline 11 & 189 & 73 & 7 & 8 & 309 & 50 & 4 & 881 & 94 \\
\hline 1116 & & 299 & 713 & 1126 & 231 & 586 & 629 & 682 & 109 \\
\hline 10 & & 14 & 411 & & 61 & 320 & 46 & 879 & 114 \\
\hline & 134 & 452 & 750 & & 195 & 206 & 427 & 745 & 111 \\
\hline 119 & 233 & 139 & 484 & 1119 & 62 & 202 & 77 & 11 & \\
\hline & 243 & 258 & 9 & & 193 & 33 & 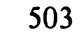 & 60 & \\
\hline 124 & & 65 & 6 & & 227 & 48 & 820 & 1139 & 112 \\
\hline 125 & & 38 & 784 & 102 & 358 & 660 & 708 & 997 & 10 \\
\hline 1 & & 70 & 5 & & 196 & 66 & 643 & 887 & 1 \\
\hline & & 579 & & & 80 & 92 & & 712 & \\
\hline & 325 & 537 & & & 197 & 35 & & 926 & 1 \\
\hline & & 1 & & & 39 & & & 38 & \\
\hline & & 726 & & & 97 & 73 & 677 & 13 & 112 \\
\hline & 511 & 20 & & & 75 & 01 & 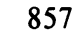 &  & . \\
\hline & 553 & 416 & & & 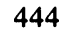 & 72 & & & \\
\hline & & 865 & & & 2 & 8 & & 33 & \\
\hline & & 4 & & & & 3 & & & 1 \\
\hline & & 1 & & & 167 & & & 33 & 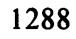 \\
\hline & & 55 & & & 46 & 79 & 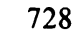 & 56 & \\
\hline & . & 12 & & & & & & 3 & \\
\hline & 383 & 1 & & & & & & & 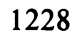 \\
\hline & 153 & 80 & & & & 4 & & & 1 \\
\hline & & 24 & & & & 1 & & & \\
\hline & & 32 & & & o & 99 & 10 & 68 & 1 \\
\hline & 181 & 709 & & & 2 & 55 & & 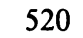 & \\
\hline & & 550 & & & 40 & 401 & 02 & 42 & ( \\
\hline & & 435 & & & & 35 & & 4 & \\
\hline & & 282 & & & 510 & & & 80 & 1 \\
\hline & & 2 & & & & & & 93 & 1 \\
\hline & & 83 & & & 3 & & & 5 & \\
\hline & & & & & & & & & \\
\hline & & 17 & & & & & & & \\
\hline & & 375 & & & 0 & 555 & 10 & 18 & 147 \\
\hline & & 290 & & & 116 & 77 & & 28 & 116 \\
\hline & 7 & 12 & & & & & & J & 70 \\
\hline & & 468 & & & & & & & . \\
\hline & 73 & 39 & & 1400 & & 4 & & & \\
\hline & 64 & 54 & 1 & 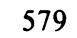 & & 8 & 442 & 365 & \\
\hline
\end{tabular}


TABLE 1 (continued)

\begin{tabular}{|c|c|c|c|c|c|c|c|c|c|}
\hline$\backslash k$ & 3 & - & 5 & $\longrightarrow$ & - & 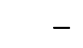 & & - & \\
\hline & & & & & & & & & \\
\hline 588 & 159 & 3 & 152 & 355 & 67 & 707 & 13 & 1116 & \\
\hline 04 & & 608 & 745 & 14 & 188 & 226 & 661 & & \\
\hline 16 & & & 836 & 11 & 75 & 15 & 421 & 499 & \\
\hline 36 & 37 & 192 & 577 & 13 & 35 & 514 & 904 & 1156 & 1 \\
\hline 20 & 397 & 23 & 1242 & & 21 & 327 & 955 & 996 & 1 \\
\hline 1652 & & 116 & 994 & 1359 & 160 & 342 & 1124 & 1302 & 101 \\
\hline 60 & 37 & 76 & 08 & & 63 & 519 & 342 & 1102 & 100 \\
\hline 74 & 755 & 149 & 600 & 1473 & 454 & 526 & 1023 & $10 / 6$ & 11 \\
\hline 692 & & 168 & 229 & 13 & 60 & 322 & 441 & 1074 & \\
\hline 24 & & 1107 & 1334 & 139 & 311 & 614 & 868 & 872 & \\
\hline 28 & & 61 & 1112 & 1582 & 72 & 648 & 701 & 850 & 140 \\
\hline 36 & & 37 & 422 & 1471 & 1109 & 1303 & 1398 & 1657 & 167 \\
\hline 744 & & 131 & 624 & 1637 & 17 & 85 & 99 & 721 & 882 \\
\hline 1756 & 99 & 351 & 1128 & & 383 & 36 & 48 & 24 & 64 \\
\hline 58 & & 477 & 958 & 1 & 23 & 51 & 613 & 739 & 06 \\
\hline$\pi$ & 607 & 775 & 1034 & 13 & 630 & 693 & 1078 & 1203 & 148 \\
\hline 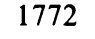 & & 4 & 287 & & & 33 & 260 & די & 456 \\
\hline 1780 & 457 & 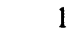 & 605 & 1173 & 623 & 37 & 656 & 970 & 1075 \\
\hline 178 & & 334 & 719 & 1249 & 43 & 661 & 1390 & 1671 & 177 \\
\hline 1788 & & 394 & 507 & 94 & 338 & 31 & 75 & 13 & 163 \\
\hline 1794 & & 315 & 562 & 10 & 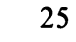 & 86 & 16 & 1 & 160 \\
\hline 1802 & 843 & 324 & 346 & 9 & 262 & 604 & 758 & 1105 & 12 \\
\hline 1 & & 627 & 1026 & 1541 & 531 & 44 & 580 & 1260 & 14 \\
\hline & & 372 & 7 & & 10 & 81 & 917 & 1293 & 16 \\
\hline & & 433 & 1545 & & 129 & 27 & 1 & 1050 & 18 \\
\hline & & & & & 7 & 06 & 4 & 72 & 16 \\
\hline 15 & 297 & 208 & 949 & 1 & 5 & 31 & 21 & 56 & 91 \\
\hline & 277 & 445 & 1492 & 1838 & 1 & 61 & 7 & 1742 & 18 \\
\hline & 113 & 98 & 1532 & 17 & & 1 & 9 & 14 & 16 \\
\hline & & 6 & 1109 & 17 & 30 & 5 & 6 & 1461 & 19 \\
\hline & & & 6 & & & 63 & & 1428 & 1530 \\
\hline & & & & & 2 & & & 1 & \\
\hline & & 2 & 673 & & 25 & 373 & 1 & 39 & \\
\hline & & 384 & 1531 & 1701 & 357 & 39 & 1204 & 1355 & 19 \\
\hline & & 109 & 1130 & & & 34 & 8 & 1379 & 13 \\
\hline & 30 & 27 & & & & 51 & 59 & 1527 & 1 \\
\hline & & & 1492 & & & & & & 905 \\
\hline & & & 398 & & & & 6 & 03 & 15 \\
\hline & & 529 & 730 & 1 & 33 & & 4 & 1468 & 15 \\
\hline & so & 54 & 7 & & 739 & 00 & 7 & 1915 & 19 \\
\hline & & & & & & & 6 & 1119 & 130 \\
\hline & & & & & & & & 48 & $78^{\circ}$ \\
\hline & 931 & 12 & & & 405 & & & 11 & 1 \\
\hline & & & & & 7 & 3 & 43 & 1279 & 19 \\
\hline & & 1 & & 566 & 6 & & 15 & 42 & 20 \\
\hline & & & & & 1552 & 34 & 1647 & 688 & 19 \\
\hline & & & & & & & & 112 & 1850 \\
\hline & 539 & & & & & & 3 & 358 & 562 \\
\hline & & & & & & ? & & 1377 & 1899 \\
\hline & 7 & & & & 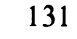 & & 78 & 1676 & 172 \\
\hline & & & & & & & & 41 & \\
\hline & 157 & 257 & & & 61 & & 51 & 1363 & 100 \\
\hline & & 918 & & & & 29 & 900 & 526 & 226 \\
\hline
\end{tabular}


TABLE 1 (continued)

\begin{tabular}{|c|c|c|c|c|c|c|c|c|c|}
\hline K & 3 & - & 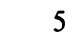 & $\longrightarrow$ & - & - & & & - \\
\hline & & & & & & & & & \\
\hline 364 & 643 & 100 & 3 & 55 & 281 & 1244 & 162 & 179 & 21 \\
\hline 70 & & 747 & 95 & & 17 & 462 & & 24 & \\
\hline 8 & 93 & 124 & 724 & & 999 & 1146 & 1478 & 86 & 173 \\
\hline 84 & & 21 & 977 & & 89 & 971 & & & \\
\hline 0 & & & & & & 39 & & & \\
\hline & & & & & & 31 & & & \\
\hline 8 & & & & & & 26 & & & 189 \\
\hline 8 & & & & & 3 & 1580 & 18 & 205 & 220 \\
\hline 02 & & 75 & 1088 & & 0 & 288 & & 1 & 203 \\
\hline 2 & & & & & 17 & 784 & & 1 & 206 \\
\hline & & & & & & 5 & & & \\
\hline & & & & & & & & & \\
\hline & & & & & & 911 & 11 & 1 & 22 \\
\hline 0 & 563 & 0 & & & J & 554 & 6 & 1376 & 268 \\
\hline 2964 & & 53 & & & 1 & 12 & & 87 & 167 \\
\hline 6 & 1087 & 95 & & & 188 & 2116 & 21 & & 0 \\
\hline & 947 & & & & & 490 & & & \\
\hline & & & & & & 13 & & & \\
\hline & & & & & & 1606 & & & \\
\hline & & & & & & 72 & & 447 & \\
\hline & & & & & 172 & 431 & 2035 & 2515 & \\
\hline
\end{tabular}

\section{BIBLIOGRAPHY}

1. J. Brillhart, D. H. Lehmer, J. L. Selfridge, B. Tuckerman, and S. S. Wagstaff, Jr, Factorization of $b^{n} \pm 1, \quad b=2,3,5,6,7,10,11,12$ up to high powers, 2nd ed., Contemp. Math., vol. 22, Amer. Math. Soc., Providence, RI, 1988.

2. S. S. Wagstaff, Jr, Update 2.6 to the second edition of factorization of $b^{n} \pm 1,1993$.

3. M. Živković, A table of primitive binary. polynomials, Math. Comp. 62 (1994), 385-386.

Institute of ApPlied Mathematics ANd Electronics, Beograd, Yugoslavia

Marka ČElebonovića 61/15, 11070 Novi Beograd, Yugoslavia 